\title{
Die Rolle des Gewohnheitsrechts im ungarischen Verfassungsrecht nach 1848
}

\section{The Role of Customary Law in Hungarian Constitutional Law after 1848}

Abstract: Even after 1848, Hungary's constitution remained a so-called 'historic constitution', a term which emphasises the central role of customary rules developed during previous centuries. The present paper aims to examine the extent to which this particular source of law persevered in certain elements of state administration and establishment. As experience shows, customary law was preserved primarily within the scope of regal authority, although during the late Middle Ages it is clearly traceable in the process of the separation of legislative and executive powers. Consequently, the daily functioning of the Parliament equally contains many rules stemming from customary law. However, the separation of administration and judiciary was processed via statutory measures for the most part, thus in this respect customary law failed to prevail.

Keywords: customary law - history of Hungarian constitution - state establishment

\section{Einleitung ${ }^{1}$}

Die Kodifikationen verschiedener Rechtszweige, die sich im 17.-19. Jahrhundert entfalteten, drängten das Gewohnheitsrecht in den Hintergrund. Sie konnten dieses zwar nicht völlig auflösen, aber die Dominanz änderte sich bedeutsam. Während vor den Kodexen das Gewohnheitsrecht bestimmend gewesen war, neben dem das Gesetz nur eine ersetzende Rolle spielte, änderte sich das nach der Ausgabe der Kodexe konträr. Der Erlass einer schriftlichen Verfassung hatte einen ähnlichen Einfluss auf die verfassungsrechtlichen Gewohnheiten. Die historische Verfassung existierte ohne gesetzliche Regeln nicht und neben einer schriftlichen Verfassung konnte auch das Gewohnheitsrecht vorkommen. Laut Móric Tomcsányi: „Die histori-

\footnotetext{
${ }^{1}$ Diese Arbeit wurde im Rahmen des Programmes "Zur Verbesserung der Qualität der Juristenausbildung" des Ungarischen Justizministeriums durchgeführt.
}

sche Verfassung schließt jedoch nicht aus, dass neben der gewohnheitsrechtlichen Entwicklung als Ergänzung auch die Gesetzgebung zur Geltung kommen kann, wie jedoch in schriftlichen Verfassungen neben den schriftlichen Rechtsquellen auch gewohnheitsrechtliche Institutionen vorkommen können. Eindeutige historische [gewohnheitsrechtliche] und eindeutige schriftliche Verfassungen existieren nicht." ${ }^{2}$ Der Schwerpunktwechsel ist jedoch aus den Worten von Tomcsányi bemerkbar: Während bei der historischen Verfassung das gesetzliche Recht nur als „Ergänzung“ existiert, kommt bei den schriftlichen Verfassungen das Gewohnheitsrecht nur noch „manchmal“ vor.

Das Zitat von Móric Tomcsányi kann noch verständlicher gemacht werden, indem dieses zusammen mit den drei Funktionen des Gewohnheitsrechts von István Werbőczy interpretiert wird: Laut ihm gäbe es gesetzerklärende, ge-

\footnotetext{
${ }^{2}$ TOMCSÁNYI, Magyarország közjoga 33.
} 
setzverderbende und gesetzersetzende Gewohnheiten. ${ }^{3}$ Das kodifizierte Recht kann nämlich nur mit dem ersten, mit der gesetzerklärenden Gewohnheit zusammenleben. Die gesetzverderbende Gewohnheit ist grundsätzlich eine schwer interpretierbare Funktion, und die Entstehung der gesetzersetzenden Gewohnheit deutet auf eine Lücke der Kodifikation hin. Das Ziel der Kodifizierung ist, eine Regelung zu schaffen, die auf die einzelnen Rechtszweige ausgedehnt jede Lebenssituation abdeckt. Falls der Richter bei einer Rechtsdebatte keine verwendbare Gesetzesregel findet, zeigt dies einen Mangel des Kodexes. Auch wenn es in einer schriftlichen Verfassung für die Lösung eines verfassungsmäßigen Problems keine verwendbare Bestimmung gibt, vermutet man eine Lücke der Verfassung. Es kann selbstverständlich vorkommen, dass manche Fragen in der verfassungsgebenden Körperschaft - wegen politischer Kompromisse - offengelassen werden, dies kann jedoch auf keinen Fall als normale verfassungsmäßige Technik betrachtet werden.

Demzufolge unterscheiden sich die zwei Verfassungstypen bezüglich der Rolle des Gewohnheitsrechts eher darin, dass sowohl gesetzersetzende als auch gesetzerklärende Gewohnheiten in einer historischen Verfassung vorkommen, im Fall einer schriftlichen Form kommt jedoch nur den gesetzerklärenden Gewohnheiten eine Rolle zu. In dieser Studie setze ich mich mit den gesetzersetzenden Gewohnheiten auseinander, weil eine Verfassung dank dieser zu einer historischen Verfassung wird.

Man soll auch einen Unterschied zwischen den kategorischen Regelungen und den Grundprinzipien der historischen Verfassung machen. Die Letzteren kommen auch im Fall des Erlasses einer schriftlichen Verfassung zur Geltung. Damit steht der Absatz (3) des Artikels R. des neuen ungarischen Grundgesetztes in Zusammen-

\footnotetext{
${ }^{3}$ WERBőCZY, Tripartitum, 11. Titel $\S \S 3-5$.
}

hang, laut dem die „Bestimmungen des Grundgesetztes [...] im Einklang mit den Errungenschaften der historischen Verfassung interpretiert werden" sollen. Die Errungenschaften (Grundprinzipien) können bei der Interpretation des Textes der Charta wichtige Richtungsangaben geben, der Text der Charta überschreibt jedoch die früheren kategorischen Regeln des Gewohnheitsrechts. Es wird sichtbar, dass zahlreiche gewohnheitsrechtliche Regeln vor 1944 im Wirkungskreis des Staatsoberhaupts oder der Regierung existierten, auch wenn es heutzutage selbstverständlich ist, dass vom Grundgesetz festgestellt wird, was zum Wirkungskreis des Staatspräsidenten bzw. der Regierung gehört. In dieser Studie werde ich unter den gesetzersetzenden Gewohnheiten vor 1944/1949 die von mir „kategorische Regeln“ genannten Elemente untersuchen. Diese sind diejenigen, die im Fall einer lückenlosen Verfassung im Rahmen einer schriftlichen Verfassung entfallen, die jedoch im Rahmen einer historischen Verfassung existieren.

Bevor die Details untersucht werden, ist es wichtig zu erwähnen, dass es nicht mein Ziel ist, und auch nicht sein kann, einen lückenlosen Katalog der gesetzersetzenden Gewohnheiten des ungarischen öffentlichen Rechts nach 1848 zusammenzustellen. Dazu wäre eine genaue Untersuchung aller verfassungsmäßigen Einrichtung nötig. Mein Ziel ist es, zu zeigen, dass den gesetzersetzenden Gewohnheiten auch nach 1848 eine wichtige Rolle zukam und diese sich auch in den Rahmen der "modernen“ Staatstätigkeit einbauen konnten. Insofern war die Verabschiedung der schriftlichen Verfassung kein unbedingt notwendiges Kriterium für die Aufrechterhaltung der europäischen Existenz.

Es ist jedoch auch selbstverständlich, dass die bürgerliche Umwandlung die einzelnen Elemente der Staatsorganisation nicht einheitlich betraf. Wo die Notwendigkeit der Umwandlung größer war, geriet die Rolle der gesetzersetzenden Gewohnheiten in den Hintergrund, und das Gesetz 
wurde zum wichtigsten Element der Regelung. Wo der Anspruch auf eine Veränderung nicht so groß war, blieben die gesetzersetzenden Gewohnheiten als bedeutende Faktoren in der Regelung bestehen.

Die folgende Frage wird hier aufgrund der Untersuchung der ungarischen Staatsorganisation nach 1848 beantwortet: In welchen Bereichen der Staatsorganisation blieben die gesetzersetzenden Gewohnheiten am meisten aufrecht? Das bedeutet sofort eine Beschränkung des Themas, da in der historischen Verfassung auch die Garantien der Grundrechte zu finden sind, man denke nur an die Goldene Bulle von 1222. Die Rechtserweiterung von 1848 bezog die Grundrechte, die bisher nur für die Adeligen galten, auf alle Staatsbürger. Auf dieses Gebiet soll hier jedoch nicht eingegangen werden.

\section{Wo blieben die gesetz- ersetzenden Gewohnheiten am meisten aufrecht?}

Wenn man also den Aufbau der Staatsorganisation nach 1848 untersucht, erfährt man, dass die gesetzersetzende Gewohnheit in ihren einzelnen Elementen nicht mit gleichem Gewicht aufrecht blieb. Vielleicht nicht ausschließlich, aber am meisten ist die gesetzersetzende Gewohnheit an die Zuständigkeiten der einzelnen Organe des Staates zu binden. Ein Staat kann so nicht existieren, dass die Organe über keinen Wirkungskreis verfügen, also mussten diese bereits bei der Entstehung des Staates existieren. Die Entstehung des Reichstags im Spätmittelalter kam aber auch grundsätzlich im gewohnheitsrechtlichen Rahmen zustande, welcher jedoch nicht nur die Frage des Wirkungskreises betraf, sondern auch eine Organisationsfrage war. Und wo es keine bedeutende Veränderung gab, blieben diese gewohnheitsrechtlichen Regelungen auch nach 1848 aufrecht.
Welche sind die staatsmächtigen Organe, deren Wirkungskreise in keinem Gesetz wurzelten, nicht einmal in der Zwischenkriegszeit? Die Einführung der Gegenzeichnung des Ministers bedeutete eine große Änderung in der verfassungsmäßigen Rechtsstellung des Königs, man kann jedoch erfahren, dass originale (gesetzersetzende) gewohnheitsrechtliche Regelungen eben hier am meisten aufrecht blieben. Die Maßnahmen des Herrschers waren nur mit der Gegenzeichnung des Ministers gültig, seine Wirkungskreise änderten sich jedoch nicht bedeutsam, sie gerieten nur unter die erwähnte Kontrolle. Fast jedes Element dieser Wirkungskreise stammte, wie später zu sehen wird, vom Gewohnheitsrecht.

In der Zusammensetzung und dem Wirkungskreis des Reichstags gibt es mehrere gesetzlichen Regelungen, es gibt jedoch auch hier ziemlich viele gesetzersetzende Gewohnheiten.

Die bürgerliche Umwandlung (1848/1867) hatte jedoch einen viel größeren Einfluss auf das System der regionalen Selbstverwaltung. So gerieten zum Beispiel die gewohnheitsrechtlichen Regelungen bezüglich der Komitate viel mehr in den Hintergrund, und die Funktion der Regelung übernahm das Gesetz hauptsächlich selbst. Ich werde kurz erwähnen, dass auch die Wirkungskreise vom Gesetz bestimmt wurden, aber die größere Menge der Gesetze zeigt auch, wie sehr das Gewohnheitsrecht in den Hintergrund geriet. ${ }^{4}$

\footnotetext{
${ }^{4}$ Es entstanden zum Beispiel bezüglich des Reichstags auch mehrere Gesetze, aber diese strebten keine ausschließliche Regelung an. Die Komitatsgesetze in der Zeit des Dualismus (GA XLII.:1870; GA XXI.:1886) waren jedoch den heutigen Gesetzen der Selbstverwaltungen viel ähnlicher. Für sie sind nicht die ergänzenden, an das Gewohnheitsrecht gebundenen Regeln typisch, sondern die detaillierte Regelung für fast jedes Gebiet der Organisation und Tätigkeit der Komitate. Solange der Art. IV der Gesetze von April bezüglich des Reichstags nur aus 15 Paragraphen bestand, umfassten beide erwähnten Komitatsgesetze
} 
Neben den Wirkungskreisen, die ja die wichtigsten Elemente sind, gab es auch anderswo gesetzersetzende Gewohnheiten. Ich habe bereits bezüglich des Reichstags erwähnt, dass der Großteil der Organisationselemente im Spätmittelalter mithilfe des Gewohnheitsrechts entstand. Die Krönung des Königs oder die Niederlegung der Krone gehören auch nicht zur Frage des Wirkungskreises, hatten jedoch einen bedeutenden gewohnheitsrechtlichen Hintergrund. Und selbst die Staatsform fußte im Gewohnheitsrecht, was noch vor der Untersuchung der Rechtsstellung des Königs in einem eigenen Teil thematisiert wird.

Bevor ich auf die konkreten gewohnheitsrechtlichen Regelungen eingehe, soll auch untersucht werden, in welcher Beziehung das Gesetz zu den gewohnheitsrechtlichen Regelungen stand bzw. wie der Reichstag mit einzelnen allgemeinrechtlichen Gesetzen das Gewohnheitsrecht gestaltete, also die gesetzersetzenden Gewohnheiten. Im Folgenden soll also diese Frage beantwortet werden, dann beginnen wir die Untersuchung der konkreten gewohnheitsrechtlichen Regelungen mit der Frage der Staatsform, und in den darauffolgenden Punkten werden die einzelnen staatsmächtigen Organe zum Thema.

\section{Das Verhältnis zwischen Gewohnheitsrecht und Gesetz}

In der Einleitung habe ich bereits erwähnt, dass das Gesetz neben dem Gewohnheitsrecht in der historischen Verfassung nur eine ergänzende Rolle hatte. Das heißt, dass der Reichstag das Mittel der Gesetzgebung zur Umgestaltung des Gewohnheitsrechts verwendete. Wann dachten die ungarischen Stände (der Reichstag), dass es

fast 100 Paragraphen. Und das war nur eine Quelle für die Regelung der regionalen Selbstverwaltungen, da es über die Gemeinde ein anderes Gesetz gab. nötig war, in einigen allgemeinrechtlichen Fällen Gesetze zu erlassen? Meiner Ansicht nach gibt es hier drei Hauptgründe dafür.

Der erste Faktor, welcher die Gesetzgebung motivierte, war die Einführung einer neuen öffentlich-rechtlichen Institution. Selbst die bürgerliche Umwandlung konnte sich mit der gewohnheitsrechtlichen Entwicklung nicht verwirklichen. Neue öffentlich-rechtliche Institutionen waren z.B. die ministerielle Gegenzeichnung oder das repräsentative Wahlrecht, welche der Reichstag durch die Gesetzgebung eingeführt hat.

Der zweite Faktor, welcher die Gesetzgebung motivierte, hatte die Verstärkung des Gewohnheitsrechts zum Ziel. Dies kam vor, wenn die Existenz einer gewohnheitsrechtlichen Norm umstritten war, was meistens im Verhältnis zwischen dem König und dem Reichstag auftrat. Entweder der Herrscher oder der Reichstag erkannte das auf Gewohnheitsrecht basierende, staatsmächtige Recht des anderen nicht an, was deshalb später, um die Spannung zwischen dem König und den Ständen zu lösen, gesetzlich festgelegt wurde. Die wichtigste Vorbedingung dafür, dass die Regelung einer Sache im Rahmen des Gewohnheitsrechts blieb, war nämlich der Konsens hinsichtlich der Regelung. Falls dieser sich auflöste, musste das Gewohnheitsrecht gesetzlich bekräftigt bzw. geklärt werden.

Der dritte Faktor, welcher die Gesetzgebung motivierte, war die Modifizierung, die Korrektur des Gewohnheitsrechts. Dies kam vor, wenn die Regel, die vom Gewohnheitsrecht gestaltet wurde, zwar nicht umstritten war, jedoch diese nicht genauso verwendet werden sollte wie früher.

Die Einführung der neuen öffentlich-rechtlichen Institution betraf ein Gebiet, welches bisher ungeregelt war, konnte aber auch die Vernichtung des Gewohnheitsrechts nach sich ziehen. Diese Elemente fallen somit außerhalb des Themas dieser Studie, da sie öffentlich-rechtliche Elemente, die auch nach 1848 aufrecht blieben, 
nicht enthalten. Die anderen zwei Faktoren beziehen sich jedoch auf unser Thema, da die gewohnheitsrechtlichen Wurzeln unberührt blieben. Aus diesem Grund werden auch die gesetzlich festgelegten Fragen an das Gewohnheitsrecht gebunden.

\section{Die Bestimmung der Staatsform}

In der juridischen (rechtshistorischen) Literatur wird nicht allzu oft betont, dass die Bestimmung der Staatsform im ungarischen öffentlichen Recht erst nach der Einführung der Republik eine deklarative Form bekam. Man trifft auf Bestimmungen bezüglich der Staatsform erst in dem Volksbeschluss vom Herbst 1918,5 später Anfang 1946 im Gesetz über die Staatsform Ungarns. ${ }^{6}$ Eine Deklaration „Ungarn ist ein Königreich" kann man nicht finden, egal wie oft man das Corpus Iuris Hungarici (die ungarische historische Gesetzsammlung) liest. Die Gesetze wiesen zwar oft bei der Benennung öffentlicher Institutionen auf die Staatsform hin, (z.B. ungarisch-königliche Staatsbahn, ungarisch-königliche Landwehr usw.) aber diese waren keine Bestimmungen über die Staatsform, sondern behandelten die gegebene Situation als Fakt. Die Staatsform wurde nämlich vom Gewohnheitsrecht bestimmt, welches so eindeutig war, dass man es sogar später für nicht wichtig hielt, diese gesetzlich festzulegen (zu verstärken).

Diese Art der Bestimmung der Staatsform ergab um die Jahreswende 1919/1920 eine interessante Situation. Nach dem Sturz der Räterepublik (August 1919) wollten die politischen Kräfte, die die Macht besaßen, nicht mit der ursprünglichen

\footnotetext{
${ }^{5}$,Ungarn ist eine von anderen Ländern unabhängige und souveräne Volksrepublik." Zit.n. Art. 1 des Volksbeschlusses vom 16. 11. 1918.

${ }^{6}$ „Ungarn ist eine Republik.“ Zit.n. § 2 Abs. (1) Gesetzesartikel I von 1946.
}

Institution des Königreichs brechen, die Entente versuchte jedoch, die republikanische Staatsform $\mathrm{zu}$ oktroyieren. Die Friedrich-Regierung konnte deswegen - in ihrer instabilen Situation nichts anderes tun, als die republikanische Staatsform eindeutig $\mathrm{zu}$ akzeptieren. Diese wurde auch in einer Regierungsverordnung verlautbart, ${ }^{7}$ aber es reicht auch, sich die Schriften der Ministerpräsidentschaft anzuschauen, in denen auf dem Registrierungsstempel die Aufschrift „Büro des Ministerpräsidenten der Republik Ungarn" stand. Die Siegermächte hatten mit der monarchischen Staatseinrichtung keine prinzipiellen Probleme, sie versuchten nur die Rückkehr der Habsburger bestmöglich zu verhindern. Wenn Ungarn Republik wird, schließt das die Thronbesteigung aus, bliebe die Staatsform jedoch das Königreich, bliebe die eventuelle Rückkehr von Karl I. (IV.) eine offene öffentlich-rechtliche (und politische) Frage. Aus diesem Grund lehnte die Entente sogar die Möglichkeit einer Volksabstimmung über die Staatsform ab. ${ }^{8}$

Bei der Vorbereitung des Gesetzes über die Wiederherstellung der Verfassungsmäßigkeit (Gesetzesartikel I von 1920) ist es deshalb kein Zufall, dass die Erwähnung der Staatsform vergessen wurde. Eine Betonung der Republik wurde aus prinzipiellen Gründen vermieden, und genauso die eindeutige Offenbarung des Königreichs aus Angst vor der Retorsion der Entente. Eine sofortige Änderung gab es weder nach dem Erlass des Gesetzes am 28. Februar,

\footnotetext{
7 „Die Gerichte richten, solange bis die Verfassungsmäßigkeit völlig wiederhergestellt wird, im Namen der Ungarischen Republik", erschienen in Budapesti Közlöny am 20. 8. 1919. Bestimmung mit der Nummer 4038/1919. ME. § 1 Abs. (2).

${ }^{8}$ Ministerpräsident Károly Huszár verwies darauf auch in der Debatte des Ministerrats über den Gesetzesentwurf bezüglich der Wiederherstellung der Verfassungsmäßigkeit. Siehe: Ungarisches Nationalarchiv, Sektion K-27 (Ministerratsprotokolle), Sitzung am 13. 2. 1920, 33.
} 
noch nach der Wahl von Miklós Horthy zum Reichsverweser am 1. März. Auf dem Registrierungsstempel stand auch in der ersten Hälfte des März die Aufschrift „Büro des Ministerpräsidenten der Republik Ungarn“. ${ }^{9}$

Die gewohnheitsrechtliche Art der Bestimmung der Staatsform machte es eigentlich nicht nötig, diese deklarativ in einem Gesetz festzulegen. Das heißt, dass es für die Verlautbarung der monarchischen Staatsform nicht nötig war, in dem Gesetzesartikel I von 1920 über die Wiederherstellung der Verfassungsmäßigkeit kategorisch festzulegen, dass Ungarns Staatsform das Königreich sei. Zweifel hätte der Volksbeschluss vom November 1918 schaffen können, der die Volksrepublik proklamierte, dessen Gültigkeit aber umstritten ist. Dieser wurde dann im Gesetz über die Wiederherstellung der Verfassungsmäßigkeit ungültig gemacht, ${ }^{10}$ ohne ein einziges Wort über die Staatsform zu erwähnen. Das war aber eigentlich auch nicht nötig. Die einzige Frage blieb nur, wann die Regierung den Mut finden würde, diese auch öffentlich zu verlautbaren. Das geschah schließlich am 18. März, als zum I. Gesetzesartikel von 1920 eine Durchführungsverordnung erlassen wurde, in der vorgeschrieben wurde, bei der Bezeichnung öffentlicher Institutionen die Adjektive „königlich“ bzw. „ungarisch-königlich“ zu verwenden. ${ }^{11}$

Es lohnt sich auch auf den einen Satz des Entthronungsgesetzes von 1921 einzugehen, welcher erklärte: „Die Nation behält unverändert die Staatsform des Königreichs. "12 Das ist jedoch keine Bekräftigung der Staatsform in einem Gesetz, sondern die Feststellung, dass die Auf-

\footnotetext{
${ }^{9}$ Ungarisches Nationalarchiv, K-26-1920-III-1443, 2160/1920. Die als Beispiel erwähnte Schrift wurde am 12. 3.1920 registriert.

${ }^{10}$ "Die sog. Volksbeschluss und Volksgesetze, registriert von dem Landesgesetzblatt, werden hiermit gelöscht." zit.n. § 9 Abs. (2) GA I:1920.

${ }^{11}$ Verordnung mit der Nummer 2394/1920. ME.

${ }^{12} \S 3$ GA XLVII:1921.
}

hebung des Rechts des Hauses HabsburgLothringen auf den ungarischen Thron keinen Einfluss auf die Staatsform habe. Die Entthronung darf also nicht so interpretiert werden, dass diese auch die Staatsform verändert hätte.

\section{Die Rechtsstellung des Königs}

Wie bereits erwähnt wurde, blieben die gesetzersetzenden Gewohnheiten unter den staatsmächtigen Organen am meisten in der Regelung der Rechtsstellung des Königs aufrecht (siehe Punkt 2). Die Einbettung dieser Regelung in das ungarische Gewohnheitsrecht ist jedoch vielschichtiger, da mehrere Regeln über die Rechtsstellung des Königs gesetzlich festgelegt zu finden sind. Und hier muss ich auf das oben bereits erwähnte Verhältnis zwischen dem Gesetz und dem Gewohnheitsrecht verweisen, laut dem die Gesetze bezüglich des Gewohnheitsrechts dessen Bekräftigung, Korrektur und Modifizierung dienten (siehe Punkt 3). Wie bereits dort erwähnt wurde, entstanden die Gesetze für die Bekräftigung des Gewohnheitsrechts meistens wegen des Konflikts zwischen dem Herrscher und dem Reichstag, da der eine das Recht des anderen bzw. auch die eigenen Pflichten bestritt. Und auch hier soll die Gegenseitigkeit betont werden, da sich diese Gesetzen nicht nur gegen das Unrecht von der Seite des Königs wehren, sondern auch an mehreren Stellen das verfassungsmäßige Recht des Königs bekräftigen. Das zeigt auch, dass das Bild in ungarischen allgemeinhistorischen Schriften entstellt ist, klarer gesagt, unverhältnismäßig Habsburggegnerisch. Das Unrecht, welches die Nation durch den König erlitt, bekommt ein größeres Gewicht, die Bekräftigung der königlichen Rechte zeigt jedoch, dass die Abstreitung der verfassungsmäßigen Rechte in Konfliktsituationen auch von der Seite des Reichstags zustande kam. Das heißt, dass auch der Reichstag Unrecht gegenüber dem König tat, weswegen der Herr- 
scher die Bekräftigung dieser Rechte auch gesetzlich festlegen wollte.

Jetzt möchte ich einige Beispiele nennen. Der Reichstag erklärte im Jahre 1791 die Trennung der gesetzgebenden und vollziehenden Gewalt, ${ }^{13}$ bzw. wurden die Regeln für die Krönung des Königs gesetzlich festgelegt. ${ }^{14}$ Im Jahre 1867 wurde weiterhin gesetzlich festgelegt, dass die Absage des Königs nur mit der Zustimmung des Reichstags gültig sei. ${ }^{15}$ Die ständische Machtverteilung wurde mit der Entstehung des Reichstags bereits im Spätmittelalter verwirklicht, ${ }^{16}$ und es darf die Behauptung auch nicht ernst genommen werden, dass die Krönung bei der Entstehung der königlichen Macht 1791 keine Rolle gespielt hätte oder dass die Krönung keine Pflicht gewesen wäre. Die Gesetze entstanden ein Jahr nach dem Tod von Josef II., somit muss man nicht weiter erklären, warum. ${ }^{17}$ Der Grund für die Verankerung der Regeln bezüglich der Absage des Königs war die Thronbesteigung von Franz Josef im Jahre 1848, vor der es zwei Absagen gab. Der neue König wollte jedoch nicht akzeptieren, dass er ohne die Zustimmung zu den Absagen seitens des Reichstags nicht sein Amt antreten durfte. ${ }^{18}$ So war es kein Zufall,

${ }^{13}$ GA XII:1791.

${ }^{14}$ GA II.-III:1791.

${ }^{15}$ GA III: 1867.

${ }^{16}$ TimON, Magyar alkotmány 593.

${ }^{17}$ Joseph II. wies die Krönung zurück, weshalb er in der ungarischen Geschichtsschreibung bloß „behuteter König“ genannt wird. Der Grund für die Zurückweisung war eindeutig, er akzeptierte nämlich die verfassungsmäßigen Beschränkungen nicht, die mit der Krönung zusammenhingen, und gründete seine Macht auf die ausschließliche Souveränität des Herrschers. Nach seinem Tod gibt es auch Beispiele dafür, dass der Reichstag einige seiner konkreten Maßnahmen für ungültig erklärte. Der GA XXXII:1791 erklärte z.B., dass nur diejenigen von ihm erteilten Privilegien gültig seien, die später von seinem Nachfolger (Leopold II.) einzeln bekräftigt wurden.

${ }^{18}$ Das Problem tauchte auch beim Verzicht auf den ungarischen Thron von Karl I. (IV.) am 13.11. 1918 wieder auf. Die Nationalversammlung im Februar dass die gewohnheitsrechtliche Regel, welche ein wichtiges öffentlich-rechtliches Element des Ausgleichs war, gesetzlich bekräftigt wurde.

Jetzt können die Beispiele auch von der anderen Seite genannt werden, wo nicht für die Bewahrung der Rechte der Nation, sondern solcher des Königs Gewohnheitsrechte gesetzlich festgelegt wurden. Der Artikel der Gesetze von April 1848, der das Ministerium errichtete und seine Verantwortung verlautbarte, begann mit der Unverletzlichkeit (der Unverantwortlichkeit) des Königs. ${ }^{19}$ Das bedeutet offenbar nicht, dass der Herrscher vor 1848 zur Verantwortung gezogen werden konnte, und dass dies erst damit beendet wurde. Der Gedanke der Entthronung war manchen radikalen Kräften jedoch nicht fremd, so wird der Grund für die gesetzliche Festlegung der Unverletzlichkeit verständlich. Im Ausgleichsgesetz (im ungarischen Delegationsgesetz) wurde das Recht für den obersten Kriegsherrn verankert. ${ }^{20}$ Diese Bestimmung

1920 hat gemeinsam mit dem Königtum als Staatsform auch die Rechtskontinuität der verfassungsmäßigen Ordnung vor 1918 anerkannt. In der Frage, ob das Recht des Herrscherhauses Habsburg-Lothringen auf den ungarischen Thron auch nach der Auflösung der Österreichisch-Ungarischen Monarchie aufrecht blieb, haben sich jedoch zwei gegensätzlichen Parteien gebildet. Deshalb konnte die Absageerklärung von Karl I. (IV.) nicht einmal in die Tagesordnung aufgenommen werden. Diese Tatsache wurde von beiden Parteien als Bekräftigung des eigenen Standpunkts interpretiert. Laut der Partei für die Habsburger blieb Karl I. (IV.) wegen der ungültigen Abdankung der ungarische König, laut der Gegner jedoch sei es unnötig, sich mit seiner Abdankung zu beschäftigen, weil er auf sein Recht auf den Thron mit der Auflösung der Monarchie verzichtet hatte.

19 "Ihre Majestät die Person des Königs ist heilig und unverletzlich.” Zit.n. § 1 GA III:1848.

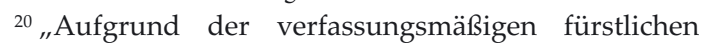
Rechte Ihrer Majestät, welche zum Wirkungskreis des Militärwesens gehören, soll alles, was sich auf die einheitliche Führung, Kommandierung und die innere Organisation der ganzen Armee und somit auch der ungarischen Armee, wie der ergänzende Teil der 
bedeutete auch nicht, dass die militärischen Rechte des Königs erst im Jahre 1867 entstanden. Ganz konkreter Grund dafür konnte das Gesetz über die Landesverteidigung sein, welches im Sommer 1848 beschlossen, jedoch von Ferdinand V. nicht sanktioniert wurde. In der Machtverteilung zwischen dem Reichstag und dem König hatte die Nation nur das Recht auf die Rekrutenbewilligung, das Organisationsrecht stand jedoch nur dem König zu. Das heißt, dass der Reichstag kein Recht hatte, die Armee tatsächlich aufzustellen oder die Befehlsrechte dem König zu entziehen. Mit der Errichtung des Ausschusses für die Landesverteidigung im Herbst 1848 wurde jedoch auch das Letztere getan.

Nach den Gesetzen, die das Gewohnheitsrecht bekräftigten, sollen im Folgenden einige Beispiele erwähnt werden, die das Gewohnheitsrecht modifizierten bzw. korrigierten. Hier geht es also darum, dass früher nicht festgelegte Regeln modifiziert, ergänzt wurden (siehe Punkt 3). Das erste Beispiel wird ein Grenzphänomen sein, welches auch im vorherigen Absatz unter den Regeln hätte vorkommen können, die das Gewohnheitsrecht bekräftigten. Das Gesetz über das Ministerium zählt mehrere Rechte des Königs auf (Auszeichnung von katholischen Erzpriestern, Begnadigungsrecht usw.), ${ }^{21}$ die der König auch weiterhin persönlich ausübt. Ich habe dieses Gesetz deshalb hier erwähnt und nicht bei den Regeln, die das Gewohnheitsrecht bekräftigten, weil es hier nicht um Rechte geht, die vorher bezüglich des Königs umstritten waren. Meiner Ansicht nach geht es hier vielmehr darum, dass der Herrscher diese Rechte nicht

gesamten Armee, bezieht, von Ihrer Majestät erledigt werden.“ Zit.n. § 11 GA XII:1867.

${ }^{21}$ „Die Auszeichnung der Erzbischöfe, der Bischöfe, der Propste und der Fahnenträger des Landes, die Ausübung des Begnadigungsrechts, die Verteilung des Adels, des Titels, des Standes, neben der Gegenzeichnung des jeweiligen ungarischen Ministers gebührt direkt Ihrer Majestät." Zit.n. § 8 GA III:1848. dem Ministerium übertragen darf. Eine andere Bestimmung dieses Gesetzes ist, dass die angeklagten Minister vom König nur im Rahmen einer Amnestie befreit werden dürfen. ${ }^{22}$ Das ist eine Modifizierung (Beschränkung) des Begnadigungsrechts. Man findet im Artikel der Gesetze von April über den Reichstag eine Regel, die das Recht für die Gesetzessanktion korrigiert (ergänzt), laut dem „die Gesetze ab dem Folgejahr von Ihrer Majestät auch während der Sitzung des Reichstages sanktioniert werden" können. ${ }^{23}$ Vor 1848 sanktionierte der König alle Gesetze nach der Schließung des Reichstags auf einmal, das heißt, dass es vorkommen konnte, dass das Ergebnis einer mehrjährigen Tätigkeit in einem Paket erlassen wurde. Nach der bürgerlichen Umwandlung (nach 1848/1867) wäre dies ein sehr langsamer Prozess gewesen, deswegen machte es die besprochene Stelle dem König möglich, dass die Beschlüsse des Reichstags vom König auch einzeln sanktioniert werden konnten.

$\mathrm{Ob}$ es ein königliches Recht gibt, welches sogar gesetzlich nicht korrigiert wurde, also ohne eine schriftliche Form nur das Gewohnheitsrecht als Quelle hatte, ist schwer zu sagen, da dazu das ganze Corpus Iuris Hungarici sehr genau gekannt werden müsste. Ich habe aber zum Beispiel das Kriegserklärungs- und Friedenabschlussrecht vor der Erwähnung des Erlasses im Gesetzesartikel I von 1920 nicht gesehen. Die Verwaltung der auswärtigen Angelegenheiten wird im Ausgleichgesetz (im ungarischen Delegationsgesetz) zwar erwähnt, aber um ganz konkrete Rechte des Königs geht es hier nicht; erst später, ähnlich wie bei dem letzten Beispiel, wird das Thema bei der Festlegung der Zuständigkeiten des Reichsverwesers wieder aufgegriffen. Es lohnt sich demzufolge, die Festlegung

\footnotetext{
22 „Im Fall eines verurteilten Ministers ist das königliche Begnadigungsrecht nur im Fall einer allgemeinen Amnestie auszuüben." Zit.n. ebd. ${ }^{23} \S 2$ GA IV:1848.
} 
der Rechtstellung des Reichsverwesers im Jahre 1920 in einem eigenen Teil zusammenzufassen.

\section{Die Festlegung der Rechtsstellung des Reichsverwesers im Jahre 1920}

Der Reichsverweser war der provisorische Vertreter des Königs, demzufolge war dessen Rechtsstellung für ihn gültig. Bezüglich seines Wirkungskreises legte der Gesetzesartikel I von 1920 über die Wiederherstellung der Verfassungsmäßigkeit das Folgende fest: „Dem Reichsverweser kommt die verfassungsmäßige Ausübung der Rechte der königlichen Macht unter den folgenden Beschränkungen zu. ${ }^{24}$ Die Beschränkungen werden dann in mehreren $\mathrm{Ab}$ sätzen aufgezählt. Die Ordnung der Regelung ist logisch, der Reichsverweser vertritt nämlich den König, übernimmt demzufolge dessen Rechte. Bei der Vertretung des Staatsoberhaupts kommt es aber oft vor, dass die Rechte des Vertreters in bestimmten Punkten beschränkt werden. Genauso hing es im Fall des im Jahre 1920 gewählten Reichsverwesers von der Entscheidung des Parlaments ab, ob dieses dem provisorischen Staatsoberhaupt jedes Recht übergab oder bestimmte Beschränkungen festlegte. Wenn es das Letztere wählte, konnte es dies offensichtlich ausschließlich auf gesetzlichem Weg tun. In Bezug auf die gewohnheitsrechtlichen Quellen stellt sich hier als allerwichtigste Frage: Wird hier ein früheres Gesetz erwähnt, aufgrund dessen die königliche Zuständigkeit beschränkt wird? Falls die Quelle der königlichen Zuständigkeit ein früheres Gesetz gewesen wäre, hätte man die Beschränkung durch die Modifizierung dieses Gesetzes erreichen können. Der Gesetzesartikel I von 1920 erwähnt aber kein solches Gesetz.

\footnotetext{
${ }^{24} \S 13$ Abs. 1 GA I:1920.
}

Bezüglich der Gesetzessanktion war zum Beispiel zu sehen, dass deren Ausübung im Jahre 1848 korrigiert wurde (siehe Punkt 5). Der Gesetzesartikel I von 1920 legte trotzdem nicht fest, dass der Reichsverweser das königliche Sanktionsrecht, welches im IV. Gesetzesartikel von 1848 festgelegt wurde, mit den folgenden Beschränkungen ausüben durfte, weil jener nicht dieses Gesetz als Quelle des Wirkungskreises betrachtete. Das Sanktionsrecht wird ohne jegliche Angabe einer gesetzlichen Quelle erwähnt, weil die Wurzel dieses Rechts im Gewohnheitsrecht lag.

Bei der Verantwortung des Reichsverwesers können wir eine ähnliche Folgerung ziehen. Es war zu sehen, dass der Gesetzesartikel III von 1848 die Unverletzlichkeit der Person des Königs festlegte (siehe Punkt 5), welche dem Reichsverweser nicht zukam. Obwohl das Gesetz die Person des Reichsverwesers auch unverletzbar machte, ${ }^{25}$ gehörte die Unverantwortlichkeit nicht dazu - das Parlament konnte ihn wegen einer Verletzung der Verfassung oder der Gesetze zur Verantwortung ziehen. ${ }^{26}$ Der Gesetzesartikel I von 1920 stellt wiederum nicht fest, dass die königliche Unverletzlichkeit, die im IV. Gesetzesartikel von 1848 festgelegt wurde, dem Reichsverweser, mit den folgenden Beschränkungen zukomme, sondern stellt ohne den Hinweis auf ein früheres Gesetz einfach die Beschränkungen fest. Die königliche Unverletzlichkeit stammt nämlich von dem Gewohnheitsrecht und nicht von dem genannten Gesetz.

Man trifft auf eine deklarative Feststellung des Wirkungskreises allein bezüglich des Hoheitsrechts der auswärtigen Gewalt, und zwar heißt es: „Der Reichsverweser vertritt Ungarn in den völkerrechtlichen Verhältnissen. Er kann Botschafter schicken und empfangen. Im Namen Ungarns darf er auf dem Weg des Ministeriums

\footnotetext{
${ }^{25} \S 14$ Abs. 1 GA I:1920.

${ }^{26} \S 14$ Abs. (2) ebd.
} 
Bund und andere Verträge mit den auswärtigen Mächten schließen [...]". ${ }^{27}$ In jedem anderen Fall ging es um die Korrektur, meistens die Beschränkung der einzelnen Rechte, hier jedoch um die Deklaration des Regierungsrechtes. Das bedeutet jedoch keine Entstehung eines neuen Wirkungskreises, sondern eine Bekräftigungsnorm des Gewohnheitsrechts (siehe Punkt 3). All das unterstützt unsere Behauptung, dass die auswärtige Gewalt früher in keinem Gesetz erwähnt wurde. Die Begründung des Gesetzes durch den Minister gibt uns eine klare Erklärung darüber, nämlich, dass das Land wichtige auswärtige Probleme lösen müsse, und in dieser Situation sei es wichtig, dass es während der Ausübung der auswärtigen Gewalt nicht einmal die kleinste Debatte geben dürfe. ${ }^{28}$

Es stellte sich eine interessante Situation auch um das Recht des obersten Kriegsherrn heraus. Das wird jedoch in einem anderen Teil thematisiert, da es sich lohnt, damit auch die öffentlichrechtliche Ordnung von 1946 zu verknüpfen.

\section{Das Recht des obersten Kriegsherrn (1920, 1946)}

Ich habe bereits erwähnt, dass das Recht des obersten Kriegsherrn vom Reichstag bestritten wurde, weshalb es im Gesetz des Ausgleichs bekräftigt werden musste (siehe Punkt 5). Diese Art der Regelung machte jedoch den Gegenzeichnungszwang des Rechts des obersten Kriegsherrn unsicher. Der Gesetzesartikel III von 1848 über das Ministerium beschrieb alles genau, und versuchte mit der langen Aufzählung von Synonymen (Verordnungen, Befehle, Bestimmungen, Auszeichnungen ${ }^{29} \mathrm{zu}$ vermeiden, dass sich irgendeine königliche Tätigkeit

\footnotetext{
27 § 13 Abs. (4) GA I:1920.

${ }^{28}$ Verhandlungen der ungarischen Nationalversammlung 22-23.

${ }^{29}$ § 3 GA III: 1848
}

der Gegenzeichnung des Ministers entziehen konnte. Die Bekräftigung des Rechts des obersten Kriegsherrn wurde im Gesetz des Ausgleichs festgelegt; das Gesetz über das Ministerium war für die Ausübung der gemeinsamen Angelegenheiten nicht gültig, darin wurde nur die Gegenzeichnung des Ministers bezüglich der Verwendung der ungarischen Armee außerhalb des Landes vorgesehen. ${ }^{30}$ Die Armee war aber nach dem Ausgleich größtenteils eine gemeinsame, die selbständige ungarische Armee machte nur einen kleinen Teil der ganzen Armee aus. Franz Josef nutzte diese gespaltene Regelung aus und das Recht des obersten Kriegsherrn wurde vom Kreis der Gegenzeichnung des Ministers herausgenommen.

Im Jahre 1920 wollte man dieses Problem lösen, deshalb legte der von der Nationalversammlung eingereichte Gesetzentwurf ausdrücklich die Erweiterung der Gegenzeichnung des Ministers auch auf dieses Gebiet fest. ${ }^{31}$ Der öffentlichrechtliche Ausschuss der Nationalversammlung hielt die Unbestreitbarkeit des Rechts des obersten Kriegsherrn für genauso wichtig wie die Verfasser des Gesetzentwurfes die auswärtige Gewalt (siehe Punkt 6). Sie empfahlen deswegen einen ergänzenden Satz, welcher exakt die Ausübung des Rechts des obersten Kriegsherrn durch den Reichsverweser festlegte, welcher letztlich tatsächlich in den Text des Gesetzes übernommen wurde. ${ }^{32}$

\footnotetext{
${ }^{30} \S 8$ ebd.

31, ,[...] All seine Anordnungen und Maßnahmen, ebenso die Anordnungen bezüglich der bewaffneten Armee sind nur in dem Fall gültig, dass diese mit der Gegenzeichnung des ungarischen Ministers versehen sind.“ Zit.n. §13 Abs. (6) Gesetzesentwurf über die Wiederherstellung der Verfassungsmäßigkeit und die provisorische Lösung der Ausübung der Staatsmacht; Verhandlungen der ungarischen Nationalversammlung 7.

$32 \S 13$ Abs. (6) des GA I:1920 wurde mit dem folgenden Text erlassen: „[...] All seine Anordnungen und Maßnahmen, ebenso die Anordnungen bezüglich der bewaffneten Armee sind nur in dem Fall gültig, dass
} 
Aus der Begründung des Ausschusses kann eine interessante Folgerung gezogen werden. Wie bereits mehrmals erwähnt wurde, wurde das Recht des obersten Kriegsherrn im Ausgleichgesetz (im ungarischen Delegationsgesetz) bekräftigt, welches jedoch mit der Auflösung der Österreichisch-Ungarischen Monarchie seine Gültigkeit verlor, was auch vom Gesetzesartikel I von 1920 konstatiert wurde. ${ }^{33}$ Im Ausschuss wurden Zweifel laut, ob nicht, wenn ein königliches Recht gesetzlich bekräftigt würde und dieses Gesetz später seine Gültigkeit verlöre, nicht das Recht selbst strittig würde? Die Antwort ist eigentlich verneinend, das heißt, deswegen löst sich das königliche Recht nicht auf. Da die Situation kritisch war, meinte der Ausschuss, dass es doch besser wäre, dieses Recht wieder ins Gesetz einzuschreiben. Im Bericht des öffentlichrechtlichen Ausschusses ist diese Folgerung ganz klar zu finden. ${ }^{34}$

diese mit der Gegenzeichnung des ungarischen Ministers versehen ist. Das betrifft jedoch diejenige Rechte des Reichsverwesers bezüglich des Militärswens nicht, die sich auf die Führung, Kommandierung und innere Organisierung beziehen."

${ }^{33} \S 11$ GA I:1920.

34 ,Zwar in der Bestimmung des $\S 13$, die besagt, dass die verfassungsmäßige Ausübung der königlichen Rechte mit der Ausnahme der taxativen aufgezählten Beschränkungen dem Reichsverweser gebühren, beinhaltet, dass die Ausübung der Rechte, die früher dem Staatsoberhaupt versichert wurden, bezüglich der Armee auch zu dem Wirkungskreis des Reichsverwesers gehören, jedoch mit Rücksicht darauf, dass der Entwurf den § 11 aus dem GA XII:1867, in dem $\S 11$ den verfassungsmäßigen Wirkungskreis des Königs bezüglich der Armee festlegt, außer Kraft setzt, fand der Ausschuss mit besonderem Hinblick auf diejenigen wichtigen nationalen Interessen, die sich mit der starken Organisation der nationalen Verteidigung und mit deren ungehinderter Arbeit in der schwierigen Situation des Landes gebunden sind, nötig, um alle Zweifel aufheben zu können, sich ganz klar und entschlossen zu äußern, dass diejenige verfassungsmäßige fürstliche Rechte, die laut unserer Verfassung im Wirkungskreis der Armee dem König gebühren, auch dem Reichsverweser gebühren wer-
An diesem Punkt lohnt es sich auch auf die öffentlich-rechtliche Regelung von 1946 hinzuweisen. Können die Rechte des Präsidenten der Republik an die frühere königliche Macht gebunden werden, oder bedeutete die Einführung der Republik eine völlig neue Epoche? War also die Quelle des Rechts des Staatspräsidenten ausschließlich der Gesetzesartikel I von 1946, oder konnte er Anspruch auf frühere königliche Rechte erheben, die darin zwar nicht erwähnt wurden, deren Wurzel aber im früheren Gewohnheitsrecht vorzufinden waren? Die Bestimmungen des Gesetzes waren grundsätzlich konstitutiver Art, das heißt, die Wirkungskreise des Staatspräsidenten - anders als beim I. Gesetzesartikel von 1920 - wurden nicht aufgrund der Neuregelungen bestimmter früheren Kompetenzen zusammengestellt, sondern selbst aus dem Gesetz entwickelt. Manchmal kommen jedoch einige Satzteile vor, die diesem Prinzip nicht entsprechen, wie z.B. der folgende: „Der Präsident der Republik ist zur Verwendung der ungarischen Armee außerhalb des Landes nur nach der vorherigen Zustimmung der Nationalversammlung berechtigt." ${ }^{35}$ Es ist bemerkbar, dass der Gesetzestext nach Kompromissen entstand, z.B. wollte die Partei der kleinen Landwirte die Wirkungskreise des Staatspräsidenten stärker an die frühere königliche Macht binden, z.B. wollte sie beim Befehligen der Armee dem Staatspräsidenten zwar nicht das Recht des obersten Kriegsherrn geben, aber ihm doch größere Kompetenzen gewähren. ${ }^{36}$ Der sozialde-

den." Zit.n. Bericht des Öffentlich-Rechtlichen Ausschusses der Nationalversammlung [...], in: Verhandlungen der ungarischen Nationalversammlung 30.

${ }^{35} \S 11$ Abs. (2) GA I:1946.

${ }^{36}$ "Der Präsident übt das Recht der Hauptkontrolle durch den Landesverteidigungsminister über die ungarische Armee aus, und er ernennt den Oberbefehlshaber der Armee." Gesetzesentwurf über die Praxis der staatlichen Hauptmacht (Entwurf der Unabhängigen Partei der kleinen Landwirte) §10 Abs. (5), zit.n. FöGLEIN, Államforma 213. 
mokratische und kommunistische Plan äußerte sich diesbezüglich jedoch nicht. Mit diesem kleben gebliebenen Satz wurde die Regelungstechnik von 1920 verwendet. Bei dem Reichsverweser wurde nämlich das Recht des obersten Kriegsherrn mit dieser Regelung beschränkt. ${ }^{37}$ Somit ist es auch keine unbegründete Folgerung, dass der Staatspräsident an die Regelungen vor 1945 bezüglich des Befehligens der Armee gebunden war.

\section{Die Zusammensetzung des Reichstags}

Der Artikel V der Gesetze von April 1848 über „Die Wahl der Reichstagsabgeordneten durch die Volksvertretung“ gestaltete die Zusammensetzung des Unterhauses völlig um, das heißt, dass die Zusammensetzung des Reichstags fortan gesetzlich geregelt war. Wenn später die Zusammensetzung des Reichstags (das Wahlrecht) modifiziert wurde, wurde dies immer mit einer Modifizierung des Gesetzes vorgenommen. ${ }^{38}$

Im Fall des Oberhauses (Herrenhauses) war die Situation jedoch anders. Zwar regelten der Gesetzesartikel I von 1608 bzw. der Gesetzesartikel

\footnotetext{
37 „Für die Abgabe einer Kriegserklärung oder die Verwendung der Armee außerhalb der Landesgrenze und den Friedensabschluss ist die vorherige Zustimmung der Nationalversammlung nötig." § 13 Abs. (5) GA I:1920. Wenn man sich den ganzen Text des § 11 Abs. (2) Gesetzesartikel I von 1946 anschaut, nimmt die Ähnlichkeit noch zu: „(2) Der Präsident der Republik ist zur Abgabe einer Kriegserklärung oder zur Verwendung der Armee außerhalb der Landesgrenze und zum Friedensabschluss nur im Fall einer vorherigen Zustimmung der Nationalversammlung berechtigt."

${ }^{38}$ Der GA XXXIII:1874, welcher die Novelle des Wahlrechts war, trug den folgenden Titel: „Über die Modifizierung und Ergänzung des Gesetzesartikels V und des Gesetzesartikels II von Siebenbürgerland aus dem Jahre $1848^{\prime \prime}$
}

X von 1687 die Zusammensetzung des Herrenhauses ziemlich detailliert, trotzdem erwähnt der Gesetzesartikel VII von 1885 über die Modifizierung der Organisation des Herrenhauses das nicht. Das heißt, dass die Quelle für die Zusammensetzung des Herrenhauses im Jahre 1885 vielmehr im Gewohnheitsrecht gesehen wurde als in den erwähnten Gesetzen. Demzufolge können die Regelungen von 1608 und 1687 vielmehr als eine Bekräftigung des Gewohnheitsrechts betrachtet werden als eine Regelung, die eine neue öffentlich-rechtliche Institution eingeführt hat (siehe Punkt 3). In späteren Gesetzen (aber noch vor 1885), die sich mit der Zusammensetzung des Herrenhauses auseinandersetzten, werden diese Gesetze auch nicht erwähnt. Als Beispiel kann die Aufnahme der orthodoxen Bischöfe ins Herrenhaus im Jahre 1792 erwähnt werden, ${ }^{39}$ oder das Gesetz über die kroatische Autonomie nach dem Ausgleich, in denen irgendwelche früheren Gesetze keine Erwähnung finden. ${ }^{40}$

Die Situation ändert sich aber nach 1885. Als Beispiel kann das Gesetz über die Bekräftigung der Begründung des griechisch-katholischen Bistums in Hajdúdorog erwähnt werden, welches besagt: „Die Begründung des griechischkatholischen Bistums in Hajdúdorog wird hiermit gesetzlich festgelegt, und der jeweilige Bischof dieses Bistums wird laut § 7 Gesetzesartikel VII von 1885 mit dem Recht auf die Mitgliedschaft im Herrenhaus ausgestattet." ${ }^{41}$ Hier geht es eindeutig um die Ergänzung des Gesetzes von 1885, das Recht auf die Mitgliedschaft im Herrenhaus stammt nicht vom Gewohnheitsrecht, sondern vom Gesetz. Die Situation hat sich nach der Aufstellung des Oberhauses in der

\footnotetext{
${ }^{39}$ GA X:1792.

40 "Von Kroatien, Slawonien und Dalmatien werden in das Herrenhaus des gemeinsamen Reichstags auch zwei Vertreter vom eigenen Landtag geschickt." § 36 GXXX:1868.

${ }^{41}$ GA XXXV:1913.
} 
Zwischenkriegszeit (Gesetzesartikel XXII von 1926) auch nicht geändert. Wenn die Zusammensetzung des Oberhauses verändert wurde, wurde es immer mit einer Modifizierung dieses Gesetzes vorgenommen. ${ }^{42}$

\section{Der Wirkungskreis des Reichstags, das Verhältnis der zwei Kammern}

Die zwei grundsätzlichen Wirkungskreise eines Parlaments in einem modernen Staat sind die Gesetzgebung und die Kontrolle der vollziehenden Gewalt (der Regierung). Von den beiden gehört der letztere nicht zu unserem Thema, es gab nämlich keine sich wiederholende Praxis vor 1848 dafür, dass die Abgeordneten vom Reichstag zur Verantwortung gezogen wurden, aus der sich ein gesetzersetzendes Gewohnheitsrecht hätte entwickeln können. Der Gesetzesartikel III von 1848 war ein Gesetz, welches eine neue öffentlich-rechtliche Institution einführte, welche der bürgerlichen Umwandlung diente (siehe Punkt 3). So war die Quelle für die Verantwortung des Ministers dieses Gesetz und nicht das Gewohnheitsrecht. Da die Typen der Verantwortung (rechtliche und politische) nicht geklärt wurden, gab es diesbezüglich auch manche gewohnheitsrechtliche Regel. Zwar standen das Institut des Misstrauensantrags und die Abdankung der Regierung diesbezüglich nicht im Gesetzesartikel III von 1848, trotzdem war dieses im ungarischen Gewohnheitsrecht ein existierendes Institut. Es stellte sich weiterhin auch aus dem Gewohnheitsrecht heraus, wie die Ausübung dieses Wirkungskreises zwischen

\footnotetext{
${ }^{42}$ Der GA XXII:1942 über die Neuregelung der Zusammensetzung des Oberhauses trug z.B. den folgenden Titel: „Über die Modifizierung und Ergänzung des GA XXII:1926 vom Oberhaus des Reichstags $[\ldots] . "$
}

den zwei Kammern verteilt wurde. ${ }^{43}$ Das war jedoch keine gesetzersetzende, sondern eine gesetzerklärende Gewohnheit. Die Verantwortung des Ministers stammt vom Gesetz, die Gewohnheit machte seine Verwendung konkreter. Die Tatsache der politischen Verantwortung wurde auch von der Praxis bewiesen. Die Regierung stellte sich im Abgeordnetenhaus nach ihrer Ernennung jedes Mal vor und der Ministerpräsident machte das Programm der Regierung bekannt. Die Wahl im Jahre 1905 brachte den Sieg der Kräfte mit sich, die gegen den Ausgleich waren, und als Antwort darauf hat Franz Joseph als Ministerpräsident einen General ernannt (Baron Géza Fejérváry). Die Vorstellung der Regierung kam in der Sitzung am 21. Juni 1905 zustande, in der aufgrund des Vorschlags von Ferenc Kossuth, dem Sohn von Lajos Kossuth, der folgende Beschluss erlassen wurde: „Da die Art der Voraussetzungen für eine parlamentarische Regierungsform, die an den Ministerpräsident gerichtet und von ihm in einer königlichen Handschrift gegengezeichnet und festgestellt wurde, der Regierung Fejérváry nicht entspricht, empfindet das Abgeordnetenhaus gegen die Regierung Fejérváry Misstrauen." ${ }^{44}$ Die Effektivität war jedoch noch nicht perfekt und Fejérváry blieb trotz der Annahme des Misstrauensantrags bis zum April des folgenden Jahres im Amt. Dieses Recht des Abgeordnetenhauses wurde jedoch nicht einmal von der Regierung bezweifelt, sie versuchte nur seiner Umsetzung vorzubeugen. ${ }^{45}$

\footnotetext{
${ }^{43}$ NAGY, Magyarország közjoga 321; BöLÖNY, Magyar közjog 181-182; BÖLÖNY, Magyarország kormányai 47; FERDINANDY, A magyar alkotmányjog 391-392; TOMCSÁNYI, Magyarország közjoga 477-478. ${ }^{44}$ Verhandlungen des Abgeordnetenhauses 466-468. ${ }^{45}$ Selbst der Beschluss entstand unter ziemlich „kämpferischen“ Umständen. Der Ministerpräsident überreichte nämlich dem Präsidenten des Abgeordnetenhauses noch vor dem Beginn der Debatte einen neuen Brief, in dem - wie es auch ungeöffnet jeder wusste - der königliche Erlass von der Vertagung des
} 
Wenn aber der Wirkungskreis der Gesetzgebung des Reichstags untersucht wird, ist die Situation bereits anders. Im heutigen ungarischen Grundgesetz werden die Wirkungskreise des Parlaments ganz kategorisch aufgelistet, u.a. kann man dort den folgenden Satz finden: „welcher die Gesetze gibt". ${ }^{46}$ In dem Gesetze von April 1848 gab es einen selbständigen Artikel über den Reichstag, ${ }^{47}$ aber eine ähnliche Feststellung seiner Wirkungskreise gab es nicht. Trotzdem gab es keinen Zweifel daran, dass es zum Wirkungskreis des Reichstags gehörte, die Gesetze zu geben. Bezüglich des Wirkungskreises des Königs wurde als korrigierende Regel für das Gewohnheitsrecht erwähnt, dass „die Gesetze ab dem Folgejahr von Ihrer Majestät auch während der Sitzung sanktioniert werden können“48 (siehe Punkt 5). Das ist zwar ein Hinweis auf den Wirkungskreis des Reichstags als Gesetzgeber, es wird dieser jedoch nicht deklariert, sondern macht das dazu gehörende königliche Sanktionsrecht konkreter.

Das verfassungsmäßige Verhältnis der zwei Kammern wurde gesetzlich auch nicht festgelegt. Als die Zusammensetzung des Herrenhauses im Jahre 1885 neu geregelt wurde, beschloss das Gesetz bezüglich des Wirkungskreises: „Der Wirkungskreis des Herrenhauses bleibt unverändert, die gleiche Praxis bleibt auch für die

Parlaments stand. Es entstand eine Debatte bezüglich der Tagesordnung darüber, ob der Brief sofort geöffnet werden sollte, oder erst nachdem der Misstrauensantrag diskutiert worden war. Wäre der Brief sofort geöffnet worden, wäre die Diskussion nicht zustande gekommen, da die königliche Vertagung nach der Verlesung sofort in Kraft trat, und bis zu ihrem Ablaufen konnte das Parlament nicht tagen. Der Präsident des Abgeordnetenhauses öffnete den Brief nicht, worauf der Ministerpräsident den Sitzungsraum demonstrativ verließ. War aber die Bekanntmachung des königlichen Erlasses tatsächlich verschiebbar?

${ }^{46}$ Grundgesetz Ungarns, Punkt b) Art. 1 Abs. (2).

${ }^{47}$ GA IV:1848.

${ }^{48} \S 2 \mathrm{ebd}$.
Initiativen $[\ldots]^{\prime \prime} .{ }^{49}$ Das heißt, dass das verfassungsmäßige Verhältnis der zwei Kammern gesetzlich nicht festgelegt wurde, es wurde im Jahre 1885 nur auf die durch Gewohnheitsrecht entstandenen Regeln hingewiesen. Man kann eigentlich auch nicht sagen, dass das frühere Gewohnheitsrecht gesetzlich bekräftigt wurde, da die kategorische Regel nicht festgelegt wurde, es wurde nur darauf hingewiesen, dass alles so bleibe wie bisher. Man findet im Gesetz von 1937 über die neue Feststellung des Wirkungskreises des Oberhauses: „Der Wirkungskreis des Oberhauses, falls es das Gesetz nicht anders vorschreibt, bleibt der gleiche, wie es der des Herrenhauses war. “50

\section{Die regionale Selbstverwaltungen}

Wie bereits erwähnt wurde, gehörte die Regelung der regionalen Selbstverwaltungen zu dem Bereich, in dem die gesetzersetzende Gewohnheit am meisten in den Hintergrund geriet, und wo die Gesetze zur Hauptquelle des Rechts wurden (siehe Punkt 2). Wenn von diesen z.B. das Munizipium untersucht wird, sieht man, dass bei der Feststellung der Wirkungskreise noch auf frühere Rechte hingewiesen wird: „Die Munizipien werden auch in der Zukunft im Rahmen der Gesetze die Folgenden ausüben: a) die regionale Selbstverwaltung [...]". ${ }^{51}$ Der Wirkungskreis seiner einzelnen Organe werden jedoch genauso kategorisch aufgezählt wie in einer schriftlichen Verfassung. Man findet bei der Feststellung des Wirkungskreises der Generalversammlung eine kategorische Aufzählung der Wirkungskreise von 16 Punkten,,52 beim Wirkungskreis des Obergespans von mehr als

\footnotetext{
49 § 13 GA VII:1885.

${ }^{50} \S 1$ GA XXVII:1937.

${ }^{51} \S 2$ GA XXI:1886.

$52 \S 47$ GA XXI:1886.
} 
ein Dutzend, ${ }^{53}$ beim Untergespan von mehr als 20 Punkten. ${ }^{54}$

Bei der Festlegung der Wirkungskreise der Gemeinde gibt es sogar keine Verweisung auf frühere Rechte ${ }^{55}$ und man darf den bereits erwähnten Fakt nicht vergessen, dass es sich hier um Gesetze handelt, deren Texte viel länger sind als es bei anderen Gesetzen mit gewohnheitsrechtlichen Inhalt typisch ist (siehe Punkt 2). Diese zeigen eindeutig, dass das Gewohnheitsrecht in den Hintergrund gestellt wurde.

\section{Epilog}

In dieser Studie wollte ich die Wurzel der Rechtsquelle der historischen Verfassung Ungarns untersuchen, darunter auch erstens die Grenzlinie, die diese von der schriftlichen Verfassung unterscheidet. Diese waren die Rolle und Existenz der gesetzersetzenden Gewohnheiten. Man kann feststellen, dass diese auch nach der bürgerlichen Umwandlung (nach 1848) fähig waren, die Staatsorgane problemlos zu bedienen, sie hatten oft sogar genauere, eindeutigere Regeln als der Text einer schriftlichen Verfassung. Es lohnt sich, sich anzusehen, was für eine große Debatte es in der Fachliteratur in der Zwischenkriegszeit über die Interpretation der gesetzersetzenden Gewohnheiten gab, und was für eine Interpretationsdebatte es heutzutage bezüglich einiger schriftlichen Bestimmungen des Grundgesetztes gibt. Für letzteres kann statistisch gezeigt werden, wie groß die Zahl der Gegenmeinungen in der Praxis des Verfassungsgerichts ist, und wie viele weitere Gegenmeinungen in der Fachliteratur aufscheinen.

Man kann nicht sagen, dass es bezüglich der Interpretation der gesetzersetzenden Gewohnheiten keine Debatte gab, da der Hauptgrund

\footnotetext{
${ }^{53} \S 57 \mathrm{ebd}$.

${ }^{54} \S 68$ ebd.

${ }^{55} \S 21$ GA XXII:1886
}

für die Entstehung der gewohnheitsrechtbekräftigenden Gesetze genau das war. Die Analyse des Verhältnisses des Gewohnheitsrechts und des Gesetzes (siehe Punkt 3) zeigte aber, dass die Gesetzgebung die Interpretationsprobleme über das Gewohnheitsrecht gut gelöst hat. Dazu war es selbstverständlich auch nötig, dass die Macht des Parlaments bezüglich der Interpretation der Verfassung noch nicht in Frage gestellt wurde.

\section{Korrespondenz:}

Prof. Dr. István SZABÓ

Pázmány Péter Katolikus Egyetem

Jog- ès Államtudományi Kar

Szentkirályi u. 28-30

1088 Budapest,.

szabo.istvan@jak.ppke.hu

ORCID-Nr. 0000-0001-5150-731X

\section{Abkürzungen: \\ Siehe das allgemeine Abkürzungsverzeichnis: [http://www.rechtsgeschichte.at/files/abk.pdf]}




\section{Ungedruckte Quellen:}

Ungarisches Nationalarchiv, Sektion K-27 (Ministerratsprotokolle), Sitzung am 13. 2. 1920, 33.

Ungarisches Nationalarchiv, K-26-1920-III-1443, 2160/1920. (12. 3. 1920).

István WERBŐCZY, Tripartitum opus iuris consuetudinarii inclyti regni Hungariae (1514).

\section{Literatur:}

József BÖLÖNY, Magyar közjog [Ungarisches öffentliches Recht], Bd. 2 (Budapest 1943).

József BÖLÖNY, Magyarország kormányai 1848-1992 [Regierungen Ungarns 1848-1992] (Budapest 1992).

Gejza FERDINANDY, A magyar alkotmányjog tankönyve [Lehrbuch des ungarischen Verfassungsrechts] (Budapest 1911).

Gizella FöGLEIN, Államforma és államfői jogkör Magyarországon 1944-1949 [Staatsform und Wirkungskreis des Staatsoberhauptes in Ungarn 1944-1949] (Budapest 2001).

Ernő NAGY, Magyarország közjoga (Államjog) [Das öffentliche Recht Ungarns (Staatsrecht)] (Budapest 1901).

Ákos TIMON, Magyar alkotmány- és jogtörténet [Ungarische Verfassungs- und Rechtsgeschichte] (Budapest 1910).

Móric TOMCSÁNYI, Magyarország közjoga [Das öffentliche Recht Ungarns] (Budapest 1932).

Verhandlungen des Abgeordnetenhauses des ungarischen Reichstages. Stenographische Berichte, Bd. 1 (1905).

Verhandlungen der ungarischen Nationalversammlung. Anlagen zu den Stenographischen Berichten, Bd. 1 (1920). 